\title{
EBSD Pattern Collection and Orientation Mapping at Normal Incidence to the Electron Beam
}

\author{
J.K. Farrer, M.M. Nowell, Damian J. Dingley and David J. Dingley \\ TSL/EDAX, 392 East 12300 South, Draper, Utah 84020
}

The large specimen tilt angle that is traditionally required for capturing high quality electron backscatter diffraction patterns (EBSP) in the SEM was first suggested by Alam, Blackman, and Pashley [1] and then confirmed by Venables and Harland [2]. It is now standard for commercial EBSD systems to be designed upon the premise that the normal to the specimen surface must be tilted to approximately $70^{\circ}$ with respect to the incident beam. Unfortunately, this specimen geometry creates many setbacks to traditional SEM functionality. For example, secondary-electron imaging is distorted due to the tilt and can be further impaired when variations in surface topography exist (e.g. fractured or etched surfaces). Also, quantification of chemistry based on X-ray energy-dispersive spectrometry can be problematic, and Z-contrast in backscattered electron imaging is almost entirely eliminated. Finally, issues such as specimen size and in-situ heating experiments may also be limited by the large tilt.

It was suggested by Venables et. al., and has now been shown, that better detection systems allow for the acquisition and use of EBSD patterns at lower angles of incidence [2, 3]. As part of the study reported here, a current TSL/EDAX OIM system, equipped with a Digiview 1612 Peltier-cooled CCD camera, was used to acquire, then to automatically index, high quality EBSPs at $0^{\circ}$ specimen tilt (i.e. normal incidence). The OIM system was installed on a JEOL JSM 6400 equipped with a W filament. Operating conditions of the microscope were $25 \mathrm{kV}$ and $\sim 75 \mathrm{nA}$ of beam current.

The patterns shown in Fig. 1 were acquired from a Ni alloy (a) and $\mathrm{Si}$ (b) at normal incidence using a camera exposure of 0.85 and 2.45 seconds respectively. The contrast of the bands in the patterns appears inverted to that of patterns from highly tilted specimens. Therefore, the OIM indexing software was altered to detect the bands of the inverted patterns such that the automated indexing functionality is the same as that of the original software design [4]. By binning down the camera resolution (to $4 \times 4$ ) and decreasing the exposure time, it was possible to acquire large orientation datasets of the specimen surface in a relatively short time. The maps shown in Fig. 2 were acquired from the Ni alloy (a) and polycrystalline $\mathrm{Si}$ (b) at normal incidence using a camera exposure of 0.05 and 0.09 seconds respectively. The datasets consist of 46,316 and 72,395 data points respectively with $95 \%$ or more of these points accurately indexed in both. Indexing speeds for the scans were approximately 20 points per sec. for the Ni alloy and 10 pps for the Si dataset. These preliminary data indicate that with the current OIM detection systems, it is possible to acquire and accurately index EBSPs at relatively high speeds with only slight modification to the indexing software to account for the inverted contrast.

1. M.N. Alam, et al., Proc. Royal Soc. London A221 (1954) 224.

2. J.A. Venables and C.J. Harland, Philosophical Magazine 27 (1973) 1193.

3. E. Langer, et al., Proc. 15th International Congress on Electron Microscopy. Durban, South Africa (2002) S14. 
4. S.I. Wright, Fundamentals of Automated EBSD, in Electron Backscatter Diffraction in Materials Science, A.J. Schwartz, et al., Eds. Kluwer Academic/Plenum, New York (2000) 51.

(a)

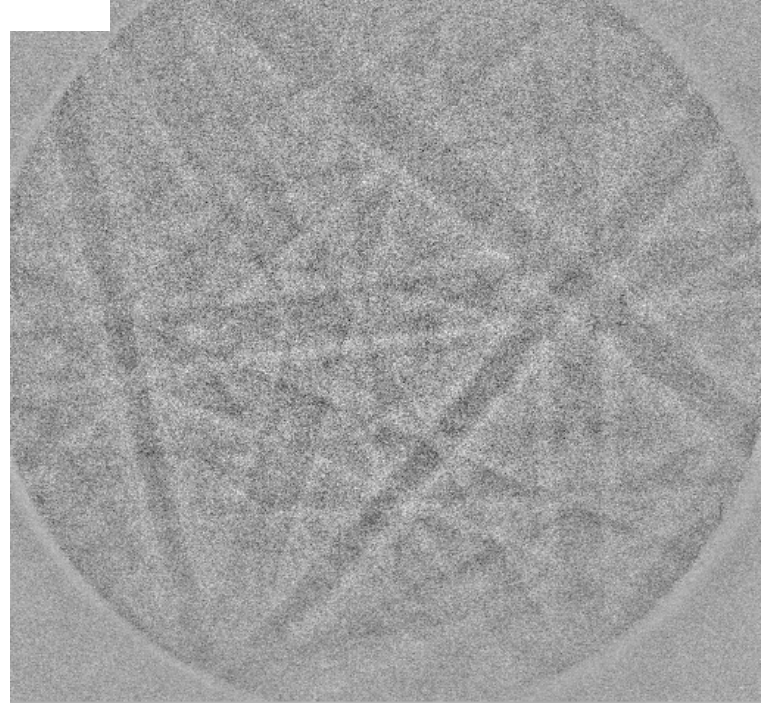

(b)

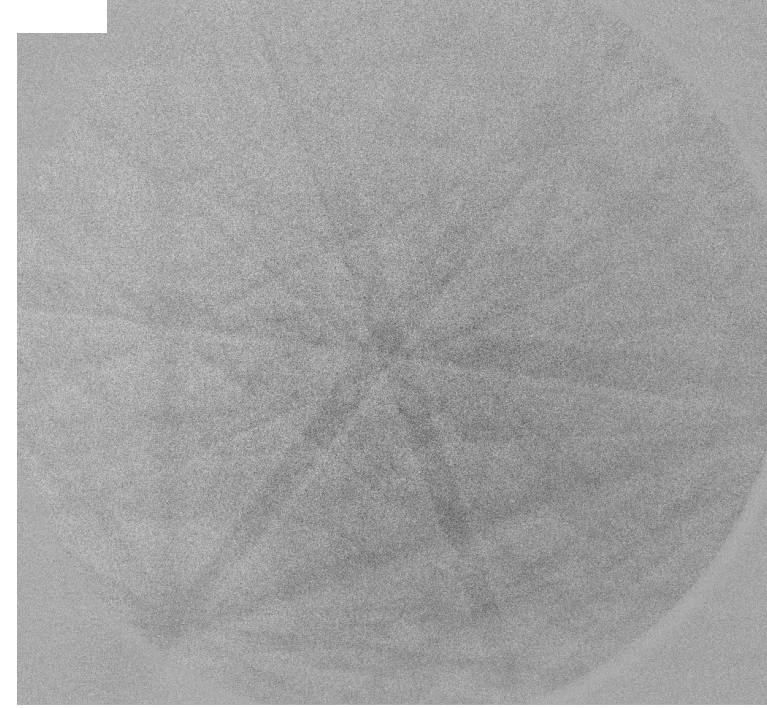

FIG. 1. EBSPs acquired from a Ni alloy (a) and $\mathrm{Si}$ (b) at normal incidence using a camera exposure of 0.85 and 2.45 seconds respectively.

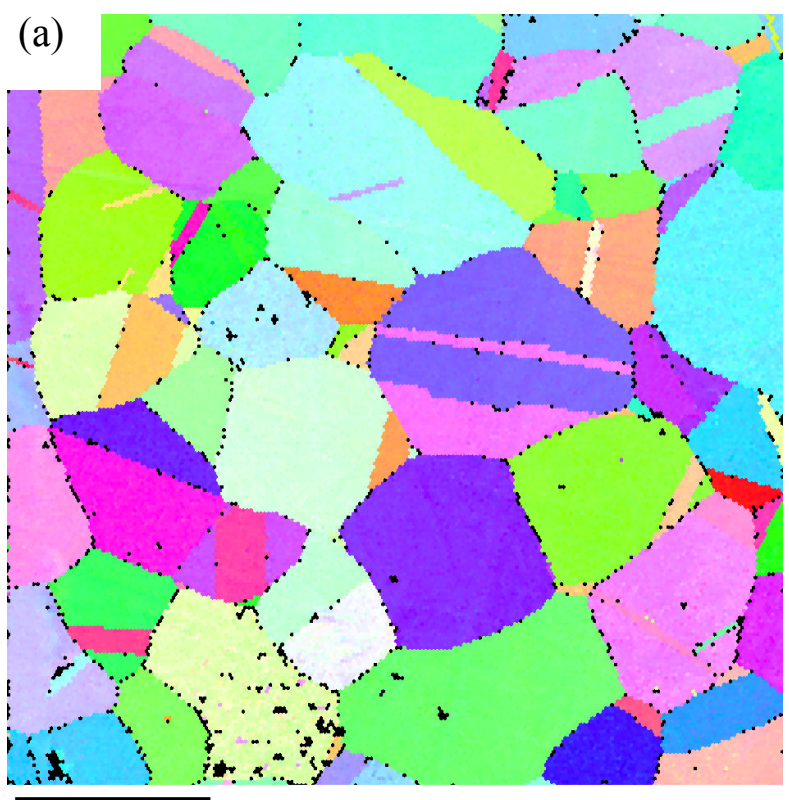

$250.0 \mu \mathrm{m}=50$ steps $\quad$ IPF $[100]$

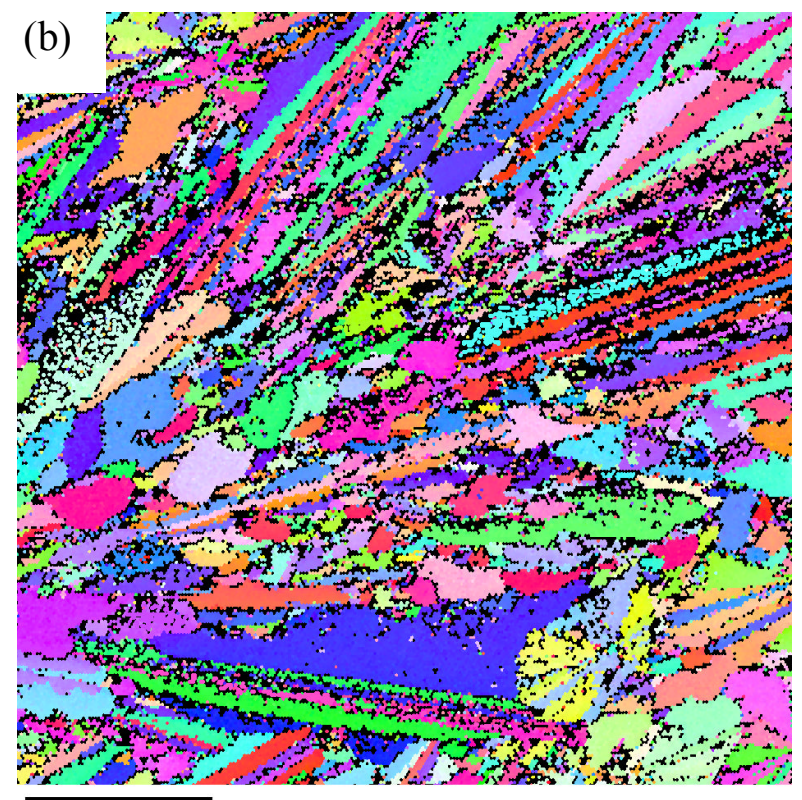

$120.0 \mu \mathrm{m}=60$ steps $\quad$ IPF [001]

FIG. 2. Orientation maps representing the dataset from a Ni alloy (a) and polycrystalline Si (b) specimens. Datasets were acquired at normal incidence using a camera exposure of 0.05 and 0.09 seconds respectively at each point in the dataset. The datasets consist of 46,316 and 72,395 data points respectively with $95 \%$ or more of these points accurately indexed in both. Points with low confidence index are displayed in black. 